\title{
Improved Technique for Recording Single Unit Activity in Awake Animals
}

\author{
THOMAS J. MORROW \\ University of Michigan. Department of Physiology, 7804 Medical Science Bldg. II, Ann Arbor, MI 48109
}

Received 22 June 1979

\begin{abstract}
MORROW, T. J. Improved technique for recording single unit activity in awake animals. BRAIN RES. BULL. 5(1)91-93. 1980.-An improved system for recording the electrical activity of single neurons in the brain of awake. unanesthetized and unrestrained animals is described. including complete details of construction and use. The advantages of this new technique over earlier methods are discussed.
\end{abstract}

Single unit activity Chronic unit recording Awake animals Moveable chronic microelectrode

IN recent years, several methods have been described for recording the electrical activity in the brain of awake animals $[1,2,4,5,6,7,9]$. While each of these methods provides certain advantages, each also has corresponding disadvantages such as allowing only one electrode track per animal, use of toxic materials $(\mathrm{Hg})$ in the system, poor stereotaxis, use uf relatively large-tip low impedance electrodes, record sampling bias for large cells, difficult construction or use, or applicable in only one species.

This paper describes a chronic moveable microelectrode system for recording extracellular potentials from single neurons in the brain of awake, behaving animals. This method offers several features which overcome some of the previously mentioned drawhacks of earlier techniques. The device is compact, relatively simple to construct and use. and allows a wide range of stereotaxic mobility and accuracy in several species.

\section{CONSTRUCTION}

The system can be divided into four parts: (1) the microelectrode, (2) the mounting sleeve, (3) the carrier tube and (4) the electrode drive screw. The microelectrode is prepared from $0.305 \mathrm{~mm}$ dia. super spring hardened-type stainless steel wire (Turotech. Inc., Stamford, CT). Ten centimeter lengths are electrolytically tapered by repeated dipping into an etching solution of one part concentrated hydrochloric acid and one part water saturated with potassium chloride using 2-8 VAC (technique modified from Green [3]). When the desired tip size and taper are obtained, the wire is sequentially immersed in water solutions of $\mathrm{NaHCO}_{3}$ (saturated), 1\% acetic acid and 95\% ethyl alcohol. Each wire is then insulated by dipping tip down into Epoxylite TM No. $600 \mathrm{M}$ lacquer (Epoxylite Corp., Buffalo, NY) and withdrawing slowly. The insulation is then cured by baking the electrode tip up in a $400^{\circ} \mathrm{F}$ oven for 15 minutes. The insulating procedure is repeated $7-8$ times. Each electrode is then tested by measuring its impedance at $200 \mathrm{~Hz}$. (technique modified from Stone [8]; microelectrodes with impedances of approximately $15 \mathrm{M}$ ohms or greater are selected for use.
The mounting sleeve ([C] in Fig. 1) is made by initially drilling a $6.350 \mathrm{~mm}$ dia. hole down the center of a $15.875 \mathrm{~mm}$ length of $9.525 \mathrm{~mm}$ dia. Delrin ${ }^{\text {T'M }}$ plastic rod, and then drilling four No. 60 holes $(1.016 \mathrm{~mm}$ dia.) through the sidewall of this tube about $1.0 \mathrm{~mm}$ from one end (see Fig. 1). A silicone rubber dental impression material (Syringe Elasticon, Kerr Sybron Corp., Romulus, MI) is then placed into the sleeve filling the side holes so that it forms a fluid-tight seal at the end of the sleeve. An additional hole is drilled through the sidewall $6.35 \mathrm{~mm}$ from the end opposite the rubber plug and is tapped for location of a $2-56$ set screw which secures the electrode carrier in the mounting sleeve.

The clectrode carrier ([B] in Fig. 1) is constructed by drilling a centered $4.763 \mathrm{~mm}$ dia. hole, $15.875 \mathrm{~mm}$ deep into one end of a piece of $6.35 \mathrm{~mm}$ dia. Delrin $7 x \mathrm{rod}, 25.4 \mathrm{~mm}$ overall length. Another hole is then made 0.5 to $1.5 \mathrm{~mm}$ off center from the opposite end to meet the larger diameter opening, and is tapped with $2-56$ threads. A third hole is made through the thickest sidewall area about $3.175 \mathrm{~mm}$ from the end so that it intersects the 2-56 threaded hole. This opening is threaded to accept a 1-72 set screw which secures the electrode drive serew during recording.

The drivescrew ([A] in Fig. 1) is made from a $28.575 \mathrm{~mm}$ length of 2-56 threaded brass rod with a No. 60 hole drilled $19.05 \mathrm{~mm}$ deep down the center. A smaller No. $78(0.406 \mathrm{~mm}$ dia.) hole is drilled on center from the opposite end of the screw to meet the larger hole. A 2-56 brass nut is threaded onto the drive $3.175 \mathrm{~mm}$ from the end in the larger hole and is soldered in place. This nut acts to limit the vertical travel of the microelectrode.

\section{IMPL,ANTATION AND USF.}

While the animal is anesthetized. a 3.0 tu $5.0 \mathrm{~mm}$ dia. craniotomy is placed above the brain region from which recording will be made. An indifferent electrode $(0.178 \mathrm{~mm}$ dia. stainless steel wire, Medwire Inc., Mount Vernon, NY) teflon TM insulated except for the cross section of the tip, is inserted into the most lateral aspect of this craniotomy to a depth of 4 to $8 \mathrm{~mm}$ into the brain and cemented in place with 


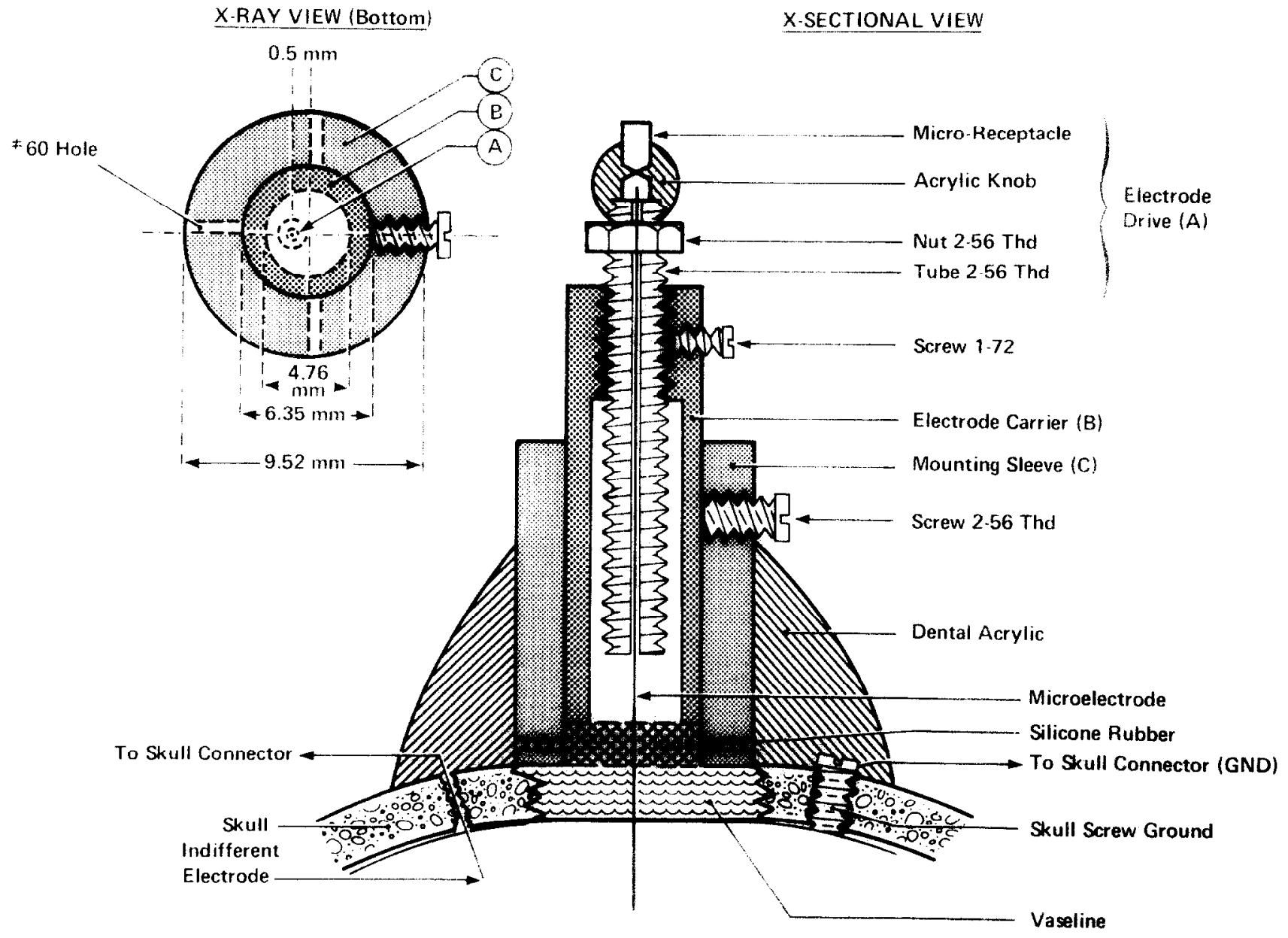

FIG. 1. Drawing of chronic microelectrode assembly (cross sectional and bottom views). Note how the silicone rubber acts to seal the cranial opening from fluid loss or contaminant entry. The Vaseline is used to stop acrylic seepage into the brain during the implantation procedure.

a cold cure dental acrylic (Perm Rebase Repair Acrylic, The Hygienic Dental Mfg. Co., Akron, OH). The chronic microelectrode mounting sleeve is then stereotaxically positioned over the craniotomy and cemented in contact with the skull by flowing dental cement around its base. A ground reference screw with wire attached is placed in the skull nearby. Gold male micro-plug contacts (Amphenol 220-P01) are crimped to the indifferent and ground wires and inserted into a plastic strip connector (Amphenol 221-1660). Dental acrylic is flowed over the wires and around the receptacle strip, completely filling the incision to its edges. During postoperative recovery, a dummy assembly consisting of a solid $25.4 \mathrm{~mm}$ piece of Delrin ${ }^{\mathrm{TM}}$ is fixed in the mounting sleeve by the set screw.

For recording. the animal is appropriately restrained using light anesthesia or other device (i.e., cat bag. primate chair, etc.) and the dummy mount removed from the sleeve. The brass drive is screwed about $4 \mathrm{~mm}$ into the Delrin ${ }^{\mathrm{Ti}}$ electrode carrier and a microelectrode inserted blunt end first through the carrier into the No. 78 hole in the end of the screw. The electrode is cut to the desired length (depending on the depth of the brain structure of interest) and after removing $2 \mathrm{~mm}$ of insulation, a gold female micro-receptacle (Amphenol 220-S01) is crimped to the electrode. The carrier assembly with the electrode tip retracted inside is placed in the mounting sleeve, turned to the desired position for the track, and secured with the setscrew. The electrode is pushed down by hand until the gold plug contacts the drive screw where it is cemented in place with dental acrylic. The acrylic forms a small knob used to advance the electrode. An FET transistor buffer circuit is plugged into the connector on the animal"s head and is also connected to the microelectrode via a wire with a male micro-plug attached. The drive is then screwed down to a position just dorsal to the intended recording site, animal restraint is discontinued and unit recording can begin. During the recording session, the microelectrode is advanced in approximately 20 to 40 micron increments while brain activity is continuously monitored. One full revolution of the drive screw gives a vertical movement of about 450 microns. All recordings are referenced to ground and the indifferent reference electrode.

lt should be noted here that during passage of the microelectrode through the silicone rubber seal, the impedance typically drops to approximately $10-15 \mathrm{M}$ ohms, depending on its initial value. This change has not been found to significantly affect the recording properties of these electrodes, in fact, tests in other laboratories have shown that metal microelectrodes with impedances of $10 \mathrm{M}$ ohms or greater pos- 


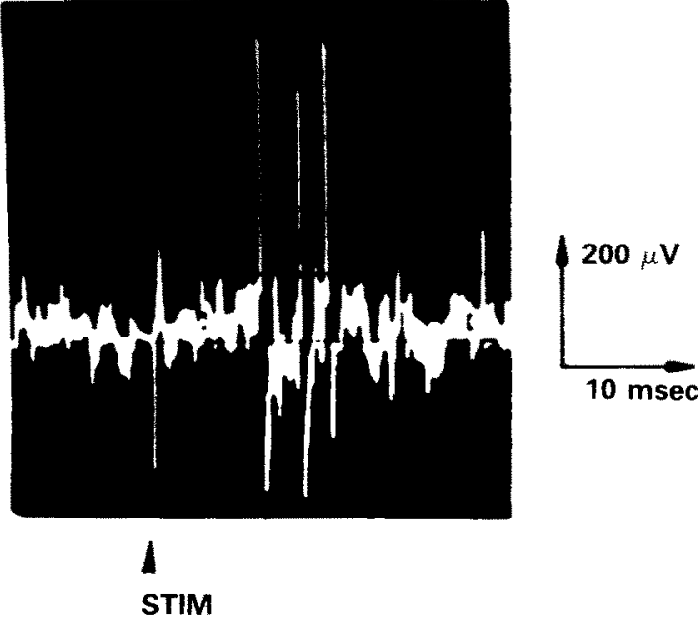

FIG. 2. Unit recorded from the nucleus Gigantocellularis of the rat in response to tail shock.

sess good recording characteristics even when sampling small cells [8]. Furthermore, microscopic examination of electrodes used in this system have shown no tip deformation or any otherwise visible damage. Figure 2 shows a sample of unit activity recorded using this system in the rat. The author has also used this technique successfully in cal and monkey.

\section{ADYANTAGES}

(1) The device is lightweight, compact and easy to use, allowing more than one assembly to be implanted in a given animal. thereby permitting one to record in multiple brain structures at the same time.

(2) Unlike other systems, the hole for the drivescrew is eccentrically located 0.5 to $1.5 \mathrm{~mm}$ off center in the carrier, allowing the electrode to be positioned anywhere on the circumference of a 1 to $3 \mathrm{~mm}$ circle by turning the plastic carrier assembly. This permits several independent and different electrode tracks to be made in the same animal.

(3) The assembly can and has been used in a variety of species including rat, cat and monkey, a leature not easily accomplished using earlier published methods.

(4) The technique permits accurate stereotaxic placement and a wide range of mobility in all planes even for deep structures. Many fine wire or rigid electrode techniques fall quite short of this capability both from the standpoint of accuracy and mobility.

(5) The relatively high impedance electrodes used in this system should reduce the apparent sampling bias towards large cells $[7]$ offering an advantage over techniques in which low impedance fine wires are used.

(6) The mounting sleeve is retrievable from the dental cement by soaking in acetone overnight, allowing the entire assembly to be reused.

\section{REFERENCES}

1. Brown, K. A., D. S. Weber and J. S. Buchwald. Chronic recording and quantification of subcortical single and multiple units. In: Brain Unit Artivity During Bohutior. edited by M. Ian Phillips. Springfield, IL: C. C. Thomas Publishers, 1973, pp. 41-52.

2. Casey, K. L. Responses of bulboreticular units to stimuli eliciting escape behavior in the cat. III. I. Netroret. 2: 19-28, 1971.

3. Green, J. A. A simple microelectrode for recording in the central nervous system. Nature 182: 962, 1958.

4. Harper. R. M. and D. J. MoGinty. A technique for recording single neurons from unrestrained animal. In: Bribu Chit Artim

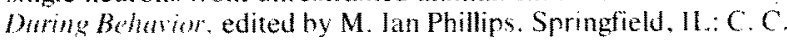
Thomas Publishers, 1973. $\mathrm{pp}$. 80-104.

5. Palmer. C. A microwire technique for recording single neurums in unrestrained animals. Brrin Res. Burll. 3: 285-289. 1977.
6. Ranck, J. B.. Jr. A moveable microelectrode for recording single neurons in unrestrained rats. In: Brain Unit Artivity During $B_{6^{\prime}-}$ harior. edited by M. Ian Phillips. Springfield, IL: C. C. Thomas Publishers, 1973, pp. 76-79.

7. Sinnamon, H. M. and D. J. Woodward. Microdrive and method for single unit recording in the active rat. Phosiol. Behat. 19: $451-453,1977$.

8. Stone. J. Sampling properties of microelectrodes assessed in the cat's retina. I. Netrophwiol. 36: 1071-1079. 1973.

9. Vertes. R. P. A device for recording single unit activity in freely-moving rats by a moveable fine wire microelectrode. $E l$ ''?-

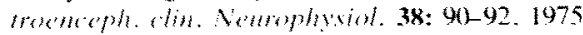

